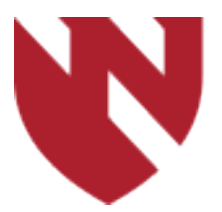

October 2021

\title{
Seropositive Pediatric Autoimmune Encephalitis: A Single Center Experience
}

\author{
Praveen Hariharan \\ University of Nebraska Medical Center \\ Daniel A. Crespo Artunduaga \\ University of Nebraska Medical Center \\ Geetanjali Rathore \\ University of Nebraska Medical Center
}

Tell us how you used this information in this short survey.

Follow this and additional works at: https://digitalcommons.unmc.edu/gmerj

Part of the Higher Education Commons, and the Medicine and Health Sciences Commons

\section{Recommended Citation}

Hariharan, P., Crespo Artunduaga, D. A., , Rathore, G. Seropositive Pediatric Autoimmune Encephalitis: A Single Center Experience. Graduate Medical Education Research Journal. 2021 Oct 04; 3(1). https://digitalcommons.unmc.edu/gmerj/vol3/iss1/13 


\section{Seropositive Pediatric Autoimmune Encephalitis: A Single Center Experience}

Creative Commons License

(c) (i) ()

This work is licensed under a Creative Commons Attribution-Noncommercial-No Derivative Works 4.0 License. 


\section{Anti-Neurofascin Antibody Associated Disease: A Case Series}

Navnika Gupta ${ }^{1}$, Afsaneh Shirani', Elizabeth Hartman'1, Lakshman Arcot'1 ${ }^{1}$ Rana Zabad ${ }^{1}$

${ }^{1}$ University of Nebraska Medical Center, College of Medicine, Department of Neurological Sciences

Mentor: Rana Zabad

Program: Neurological Sciences

Type: Original Research

Background: Autoantibodies against nodal and paranodal proteins, particularly anti-neurofascin (NF) antibodies, have been recently described in demyelinating disorders of the central and peripheral nervous system. There is an unmet need for further characterization of anti-NF associated demyelinating diseases. We describe the clinical and diagnostic findings of five patients with anti-NF antibodies, and attempt to identify features that might guide clinicians to check for them.
Methods: This is a retrospective chart review of five individuals seen at the University of Nebraska Medical Center Multiple Sclerosis Clinic who tested positive for serum anti-NF antibodies by western blot.

Results: In our series of five patients, the female to male ratio was $4: 1$, and the median age at presentation was 60 years (range: 56-70). Clinical presentations included incomplete transverse myelitis $(n=2)$, progressive myelopathy $(\mathrm{n}=1)$, recurrent symmetric polyneuropathy $(\mathrm{n}=1)$, and non-specific neurological symptoms $(n=1)$. Atypical features prompting further workup included co-existing upper and lower motor neuron features, older age at presentation with active disease, atypical spinal cord MRI features and unusual CSF findings. Serum anti-NF antibody panel was positive for the NF-155 isoform in three patients ( $\operatorname{IgM~} n=2$; IgG $n=1)$, and the NF-140 isoform in two patients $(\operatorname{IgG} n=2)$.

Conclusions: Our case-series suggests that anti-NF antibodies are more commonly detected in the older populations and women. The clinical phenotype is pleiotropic. Atypical findings on MRI and paraclinical tests prompted further workup. Larger studies are needed to assess the impact of anti-NF antibodies on long-term clinical outcomes and their associated therapeutic implications.

https://doi.org/10.32873/unmc.dc.gmerj.3.1.034

\section{Seropositive Pediatric Autoimmune Encephalitis: A Single Center Experience}

Praveen Hariharan ${ }^{1}$, Daniel A. Crespo Artunduaga', Geetanjali Rathore ${ }^{2}$

${ }^{1}$ University of Nebraska Medical Center, College of Medicine, Department of Neurological Sciences ${ }^{2}$ Children's Hospital \& Medical Center, Division of Pediatric Neurology

Mentor: Geetanjali Rathore

Program: Neurology

Type: Original research

Background: The objective of the study is to describe the clinical characteristics and identify unique features associated with antibody-positive autoimmune encephalitis (AE).

Methods: Charts of children aged below 18-years diagnosed with AE between 2005 and 2020 were reviewed. Demographics, clinical characteristics, laboratory, imaging, electrophysiological findings and long-termneurological-sequalae were collected.

Results: Among 53 AE cases, 19 had serum or CSF antibodies. Among antibody-positive
AE patients, there were $9(47 \%)$ male and 10 (53\%) female patients of whom; $10(52.6 \%)$ had anti-NMDAR-antibodies, 5 (26\%) antiVGKC-antibodies and 4 (21\%) demonstrated GAD65-antibodies. Clinical presentation included neuropsychiatric symptoms $(68.4 \%)$, altered mental status (63\%), movement disorders $(57.9 \%)$, new-onset seizures (52.6\%), speech difficulties (15.7\%), status epilepticus (10.5\%) and sleep dysfunction $(5 \%)$. Two patients $(10.5 \%)$ required ICU admission and $9(47.3 \%)$ experienced relapses needing readmissions. MRI Brain was abnormal with $\mathrm{T} 2$ hyperintensities in 13 patients $(31.5 \%)$ - 3 in parietal lobe, 2 in temporal lobe and 2 in frontal lobe. Seven patients $(36.8 \%)$ had focal EEG findings. Nine patients (47.3\%) had CSF pleocytosis and protein was normal in all patients. Acute management consisted of several different combination regimens including intravenous steroids (89\%), intravenous immunoglobulin (IVIG) (84\%), rituximab (15.8\%) and plasma exchange (PLEX) (10.5\%). Maintenance regimen included IVIG (42\%), Rituximab (36.8\%) and 1 PLEX. Six patients (31.5\%) attained complete remission and one patient underwent excision of ovarian teratoma. Long term sequalae included neurocognitive disturbances (52.6\%), epilepsy (31.5\%) and movement disorders $(5 \%)$.

Conclusion: The most frequent presentation of AE in our cohort was neuropsychiatric disturbances, followed by altered mental status. Given diverse clinical presentations, one should maintain broad differential diagnosis when evaluating pediatric patients with subacute onset neurological symptoms.

https://doi.org/10.32873/unmc.dc.gmerj.3.1.040

\section{Risk Factors for Mortality Among Patients with Gout in the Veteran's Health Administration Lindsay N. Helget, ${ }^{1,2}$ Bryant R. England,,,2 Punyasha Roul,,2 Harlan Sayles, ${ }^{2,3}$ Alison D. Petro,, ${ }^{1,2}$ Ted R. Mikuls ${ }^{1,2}$ \\ ${ }^{1}$ Veterans Affairs (VA) Nebraska-Western lowa Health Care System, Omaha, NE ${ }^{2}$ University of Nebraska Medical Center, College of Medicine, Department of Internal Medicine, Division of Rheumatology ${ }^{3}$ University of Nebraska Medical Center, College of Public Health, Department of Biostatistics}

Mentor: Ted Mikuls

Program: Rheumatology

Type: Original Research
Background: A complete understanding of factors driving the increased all-cause mortality risk in patients with gout is lacking. The purpose of this study was to examine risk factors associated with mortality among patients with gout in the Veteran's Health Administration (VHA). 